\title{
Interpreting near-side correlations and the RHIC beam energy scan
}

\author{
Paul Sorensen ${ }^{\mathrm{a}}$ \\ Brookhaven National Laboratory, Upton, NY, USA
}

Received: 20 September 2008 / Revised: 9 March 2009 / Published online: 17 April 2009

(C) Springer-Verlag / Società Italiana di Fisica 2009

\begin{abstract}
Recent data from heavy ion collisions at RHIC show strong near-side correlations extending over several units of rapidity. This ridge-like correlation exhibits an abrupt onset with collision centrality. In this talk, I argue that the centrality and beam-energy dependence of these nearangle correlations could provide access to information about the Quark Gluon Plasma phase boundary and the Equation of State of nuclear matter. A beam-energy-scan at RHIC will better reveal the true source of these correlations and should be a high priority at RHIC.
\end{abstract}

PACS 25.75.Ag $\cdot 25.75 . \mathrm{Gz}$

\section{Introduction}

Correlations and fluctuations have long been considered a promising signature for Quark Gluon Plasma (QGP) formation in heavy-ion collisions [1-4]. Early proposals for QGP searches suggested searching for a non-monotonic dependence of fluctuations on variables that can be related to the energy density created in the system-e.g. center-of-mass energy or collision centrality - the expectation being that above some energy density threshold, a phase transition to QGP would occur. The presence of the phase transition to QGP would then lead to a change in fluctuations and correlations.

These early expectations regarding finite temperature QCD are bourne out by lattice QCD calculations [5, 6]. The calculations show that for temperatures above a critical value of $195 \mathrm{MeV}$, a QGP is formed. Lattice calculations also show that baryon number, strangeness, and charge fluctuations are all enhanced near the critical temperature $T_{C}$ [7-9]. As such, correlations and fluctuations remain a topic of interest in heavy-ion collisions.

a e-mail: prsorensen@bnl.gov
Data from the experiments at RHIC indeed reveal interesting features in the two-particle correlation landscape [1019]. Specifically, it has been found that correlation structures exist that are unique to Nucleus-Nucleus collisions. While two-particle correlations in $\mathrm{p}+\mathrm{p}$ and $\mathrm{d}+\mathrm{Au}$ collisions show a peak narrow in azimuth and rapidity, the nearside peak in $\mathrm{Au}+\mathrm{Au}$ collisions broadens substantially in the longitudinal direction and narrows in azimuth. The longitudinal width of the correlation appears to depend on the $p_{T}$ of the particles. An analysis of the width of the peak for particles of all $p_{T}$ finds the correlation extends across nearly 2 units of pseudo-rapidity $\eta$ [11-19]. When triggering on higher momentum particles ( $p_{T}>2 \mathrm{GeV} / \mathrm{c}$ for example), the correlation extends beyond the acceptance of the STAR detector $(\Delta \eta<2)$ and perhaps as far as $\Delta \eta=4$ as indicated by preliminary PHOBOS data [11-19]. Furthermore, STAR has found that these correlations show an abrupt onset as a function of centrality [10]. Comparing measurements at 200 and $62.4 \mathrm{GeV}$, STAR has shown that the onset happens at the same value of transverse particle density for the different energies, suggesting the onset of the long range correlations may be related to a critical energy density.

\section{The ridge}

The ridge is a long-range correlation unique to $\mathrm{A}+\mathrm{A}$ collisions that exhibits an abrupt onset with increasing collision centrality. Phrased this way, we could conclude that this is the long sought after "smoking gun" of QGP formation. But the excitement one would expect from such a discovery has been tempered due to conflicting interpretations of the nature of these correlations. Disagreement exists as to whether the correlations are related to QGP formation or whether they in fact disprove the existence of a thermalized medium. Questions surrounding the ridge-like correlations include: are the correlations related to non-perturbative multi quark or gluon effects on minijets in $\mathrm{Au}+\mathrm{Au}$ collisions [22]? Are 

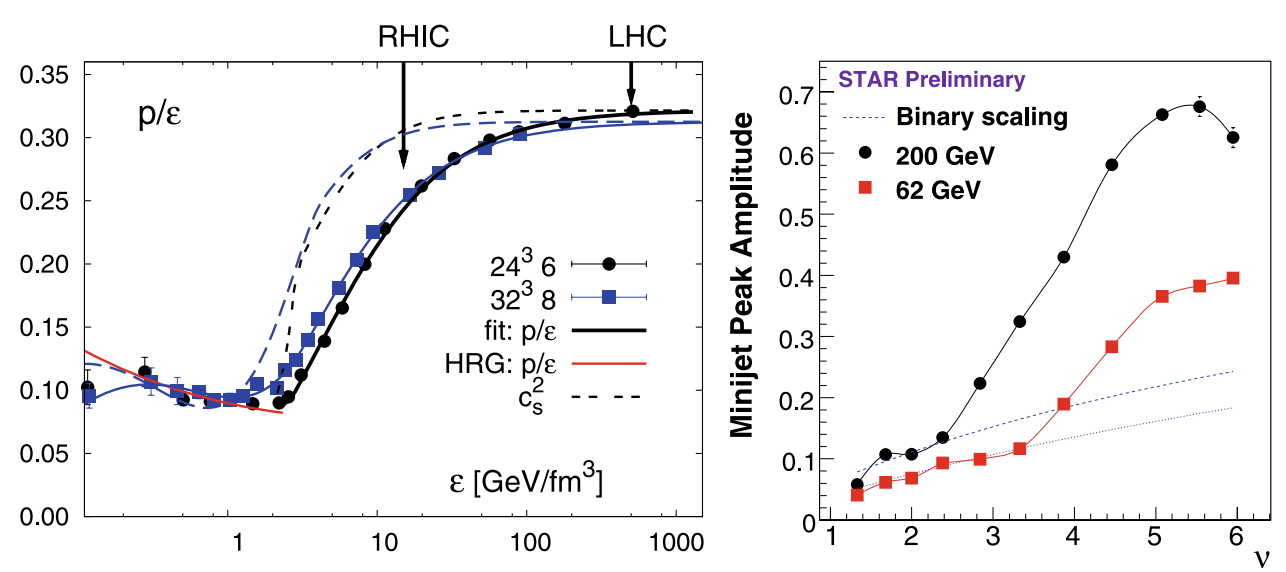

Fig. 1 Left panel: Lattice QCD calculations of the QCD equation of state. Right panel: A preliminary STAR figure showing the amplitude of the near-side ridge-like peak (described sometimes as a mini-jet peak) as presented in $[20,21]$ by special permission from the STAR

they related to soft gluons radiated by hard partons traversing the overlap region [23]? Are they related to initial spatial correlations in the system converted to momentum-space correlations by a radial Hubble expansion [25]? To beamjets also boosted by the radial expansion [26]? Or to viscous effects [24]? And do these correlations disprove the assumption of a system thermalized over some extended region [10]? These questions still remain to be answered.

Figure 2 shows a schematic illustration of the expansion after a heavy-ion collision with an emphasis on the lumpiness of the initial conditions. If the ridge is related to the translation of spatial correlations into momentum space correlations through radial flow, as illustrated in the figure, then the onset of the ridge could be related to a rise in the pressure over energy-density at the critical energy density for QGP formation. At that energy density, the liberation of color degrees of freedom in the system could lead to an increase in the pressure which in turn could lead to the flow that makes it possible to image the underlying spatial correlations in momentum space. It's not clear that hydrodynamic models will be able to reproduce such effects and the process by which the QGP transforms initial spatial correlations into momentum space correlations could be quite different than envisioned in such models. Figure 1 shows lattice QCD calculations of the equation of state $[5,6]$ on the left and the ridge amplitude at 200 and $62.4 \mathrm{GeV}$ vs centrality measure $v=2 N_{\text {binary }} / N_{\text {part }}$ on the right. When plotted vs transverse particle density related to Bjorken energy density, STAR finds that the abrupt increase in the ridge happens at the same density for both 200 and $62.4 \mathrm{GeV}$ collisions [10]; not the same $v, N_{\text {part }}$, or $N_{\text {bin }}$ but the same $\frac{1}{S} \frac{d N}{d y}$.

Above the transition, the ridge amplitude grows faster than $N_{\text {binary }}$ scaling. One analysis finds that when measuring the correlations between the leading and subleading hadrons in an event, the area of the ridge scales with the area collaboration [10]. The near-side correlations are parameterized with a 2-D Gaussian. The amplitude of the Gaussian peak is plotted in this figure and referred to as the mini-jet peak amplitude

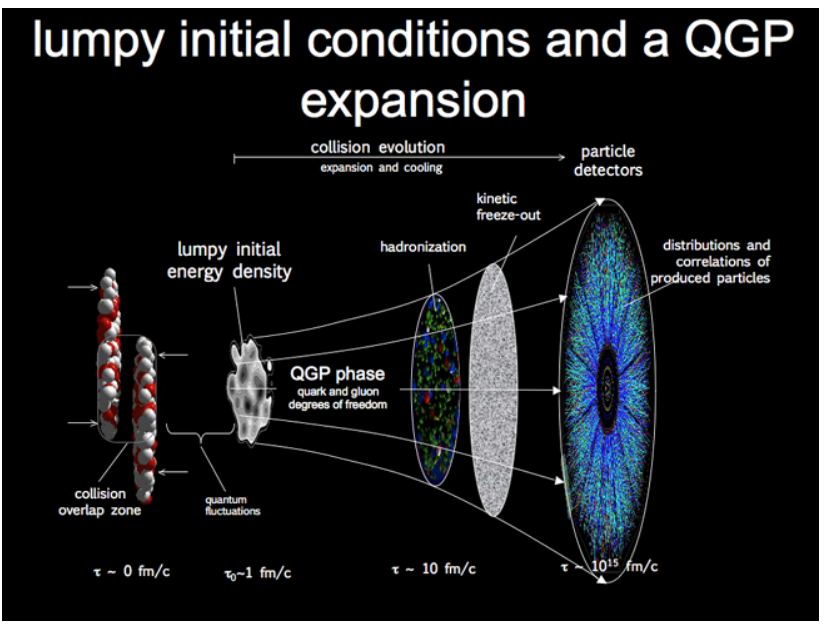

Fig. 2 A schematic diagram of the evolution of a heavy-ion collision. Possible correlations and fluctuations in the initial conditions are emphasized

of the background [27]; perhaps suggesting that the ridge correlation is related to a bulk correlation (a global correlation between all particles). It is also found that the baryon to meson ratio in the ridge is similar to the bulk and that the $p_{T}$ spectrum of the ridge is softer than for the jet-cone correlation. In fact, the only feature of the ridge that matches that of the jet cone, is that it is centered at $\Delta \phi=0$. For this reason, one may reasonably doubt whether the ridge is related to hard-scattering.

There are also observations that may prefer an explanation related to mini-jets or hard scattering. The ridge persists even when correlations are formed with trigger particles well into the fragmentation region i.e. above $p_{T}=$ $5 \mathrm{GeV} / \mathrm{c}$ where particle production is dominated by jet fragments. Also, the yield of the away-side ridge follows that of 
the near-side very closely; perhaps indicating back-to-back dijet-like correlations [10].

\section{Energy dependence}

A more extensive beam energy scan can help determine if and how the abrupt onset of the ridge is related to the onset of deconfinement. A beam-energy-scan has been proposed at RHIC and technical preparations have been made to collide beams at $\sqrt{s_{N N}}$ as low as $5 \mathrm{GeV}[28,29]$. In a recent test run, the STAR collaboration was able to gather 3000 good events at $\sqrt{s_{N N}}=9.2 \mathrm{GeV}$ after just several hours of beamtime. Collider experts anticipate increasing these event-rates by approximately a factor of 5 . The number of events required to carry out the ridge analysis shown in Fig. 1 (right) is on the order of 10 million events. These data samples can be achieved in less than a week of running for energies above $\sqrt{s_{N N}}=25 \mathrm{GeV}$. At $12.3 \mathrm{GeV}, 10$ million events will require approximately four weeks of running to acquire. This makes an energy scan of ridge phenomenology from 12.3 to $62.4 \mathrm{GeV}$ feasible at RHIC.

Dumitru et al. [20, 21] propose an explanation for the ridge based on Glasma flux-tubes. The flux-tubes themselves would not yield a narrow azimuthal correlation but if they are radially boosted, the emitted particles can be collimated in azimuth leading to a ridge like structure. This explanation of the ridge yields a prediction for the energy dependence of the ridge amplitude. For example, $\frac{\Delta \rho}{\sqrt{\rho_{\text {ref }}}} \propto$ $\frac{1}{\alpha_{s}\left(Q_{s}\right)}$ so that the amplitude of the ridge should be governed by the centrality and energy dependence of $Q_{s}$ modulated by the effectiveness of the space-momentum correlation. In $[20,21]$, blast-wave model fits were used to determine the mean flow velocity. Since the blast-wave fit parameters vary smoothly with centrality, it is not possible for that implementation of the flux-tube ridge model to reproduce the abrupt onset of the ridge. The blast-wave fits to the final hadron spectra do not necessarily accurately reflect the dynamics of the collision evolution however, so the lack of an abrupt transition in the model may simply reflect a weakness of the blast-wave parameterization and of the assumption that the QGP evolution is well described by hydrodynamics.

In Fig. 3 I plot the ridge amplitude as predicted by the model in $[20,21]$ as dashed curves. For this prediction, the following parameterization of $\beta$ is used:

$$
\begin{aligned}
\langle\beta\rangle= & \left(0.655+0.314 \log \left(N_{\text {binary }} / N_{\text {part }}\right)\right) \\
& \times\left(0.05727 \log \left(\sqrt{s_{N N}}\right)+0.2933\right) .
\end{aligned}
$$

For the solid curves, I've included an additional ad-hoc transition to illustrate how the ridge centrality and energy dependence would look if a sudden decrease in the flow occurs when the energy density in the collision drops below a
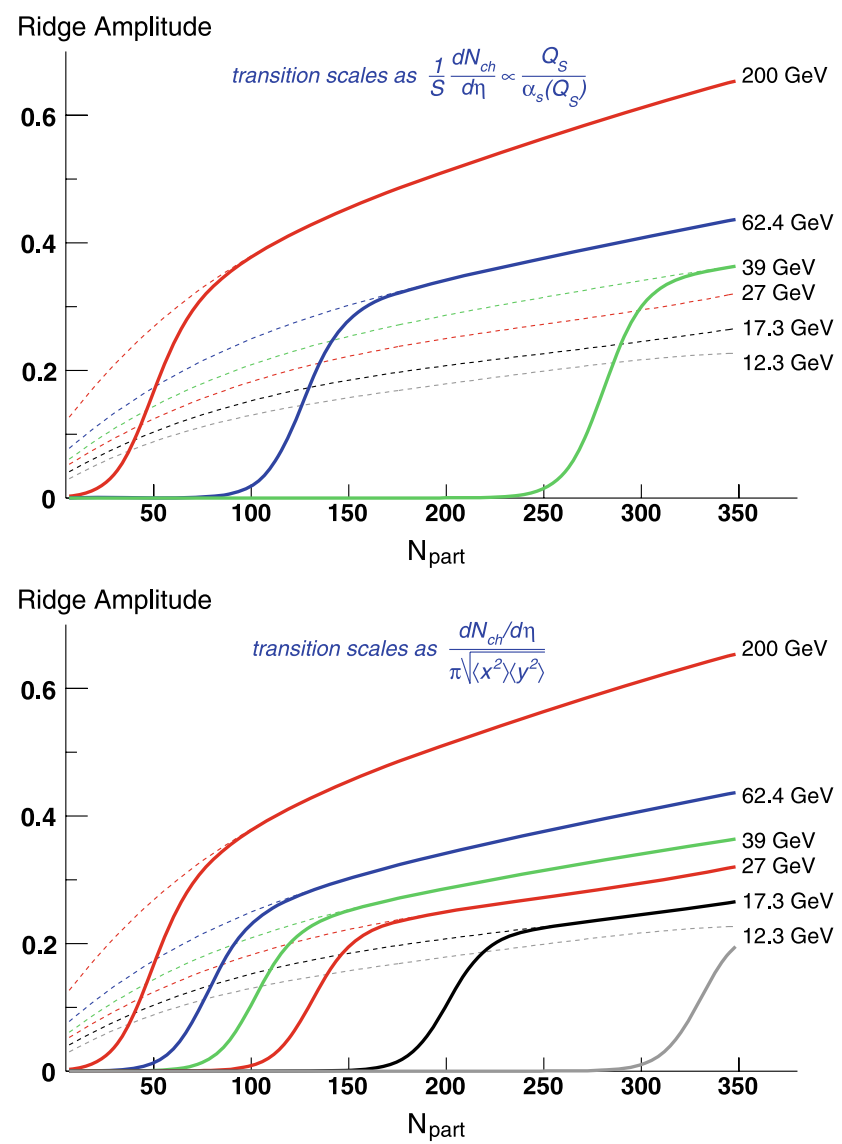

Fig. 3 The ridge amplitude from a model of glasma flux-tubes and radial flow. The dashed lines are based on flux-tubes and a blast-wave model. The solid curves in the top panel include ad-hoc abrupt transitions at fixed values of $\frac{1}{S} \frac{d N}{d y}$ as suggested by preliminary STAR measurements. The exact location of the transition at lower energies is highly dependent on the calculation of $S$ as illustrated with the alternative energy dependence shown in the bottom panel

critical energy density. This is not a prediction of the model in $[20,21]$. This decrease could perhaps be masked in data by effects from the hadronic stage. The fact that the blastwave parameterization of spectra in $\mathrm{p}+\mathrm{p}$ and peripheral $\mathrm{Au}+\mathrm{Au}$ collisions yields a non-zero value for the parameter associated with flow, does not necessarily imply flow occurs in those centralities. The parameterization used in Fig. 3, assumes that the flow "observed" with the blast-wave fit is spurious. For this transition, I follow the observation from STAR that the onset of the ridge occurs at a fixed value of $\frac{1}{S} \frac{d N}{d y}$. I then extrapolate the observed cutoff to lower energies. This transition is strongly dependent on the centrality dependence of the overlap area $S$. For this estimate I use $S=R^{2}(\theta-\sin (\theta))$ where $\theta=2 \cos ^{-1}(b / 2 R)$ and $b$ and $R$ are the impact parameter and nuclear radius respectively. In this case, the ridge persists only down to $\sqrt{s_{N N}}$ of approximately $39 \mathrm{GeV}$. Below that, the transverse density is below the minimum even for the most central collisions. Alternative calculations of $S$ such as $S=\pi \sqrt{\langle x\rangle^{2}\langle y\rangle^{2}}$ yield quite 
different results with the ridge persisting to much lower energies.

The flow velocity is only weakly dependent on energy so the variation of the ridge amplitude with energy is dominated by the change in $Q_{s}^{2} \propto\left(\sqrt{S_{N N}}\right)^{0.3}$. This gives a verifiable prediction for the energy dependence which is independent of the details of the centrality dependence. This energy dependence can be checked at LHC and in an upcoming beam energy scan at RHIC. In the case that the imaging of the flux tubes is made possible by space-momentum correlations induced by liberated color charges, then an energy scan of the ridge can be used to map out the phase boundary of the quark-gluon plasma. Given the observation of an abrupt onset of the ridge, this project should be a high priority for RHIC.

\section{Beam energy scan}

A beam energy scan has already been proposed at RHIC. One of the motivations for the energy scan is to search for signatures of the QCD critical point. According to lattice $\mathrm{QCD}$, at zero baryon chemical potential $\mu_{B}=0$, the transition from hadronic matter below $T_{C}$ to QGP above $T_{C}$ is a smooth cross-over. At higher $\mu_{B}$, model calculations indicate that the transition is a first order phase transition. The critical point lies where the smooth cross-over changes to a first order phase transition. See this list of [30-42]. Detecting the presence of the critical point depends on the ability of experiments to create matter above $T_{C}$ at larger and larger $\mu_{B}$. Accessing larger $\mu_{B}$ can be achieved by decreasing the beam energy. The question of whether the matter

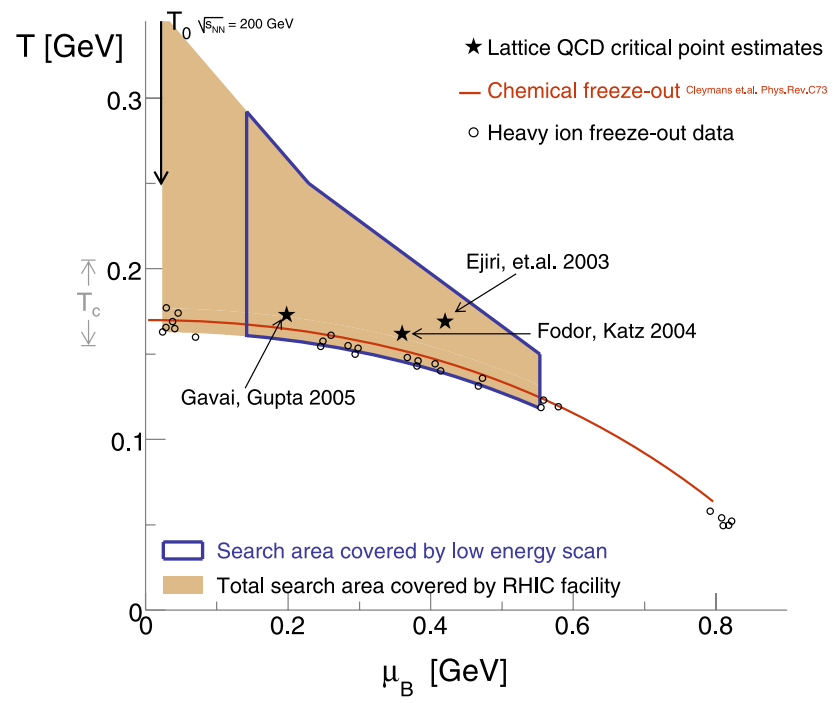

Fig. 4 A sketch of the phase diagram of nuclear matter. An estimate of the region covered by RHIC is indicated by the tan region. Three Lattice estimates for the location of a critical point in the diagram are also indicated created at high $\mu_{B}$ (low $\sqrt{s_{N N}}$ ) still reaches a temperature above $T_{C}$ is not known. Figure 4 shows a sketch of the QCD phase diagram with the region accessible in a RHIC energy scan outlined in blue. The region covered by RHIC encompasses several recent estimates for the location of the critical point from Lattice.

We note that observing correlations over a large $\Delta \eta$ range instead of short range correlations is consistent with the transition from QGP to hadrons being a smooth cross-over rather than a first order phase transition. One anticipates that a first order phase transition at freeze-out will lead to bubble formation and correlations typically smaller than $\Delta \eta=1$. Instead of these short range correlations, we observe correlations that seem to arise from the conversion of initial state, spatial correlations to momentum space correlations via strong pressure in a QGP phase. A ridge-like correlation is therefore consistent with a QGP phase followed by a smooth cross-over. The build-up of short range correlations at lower beam energies is a promising signature for a first-order phase transition. This points to the importance of large detector acceptance and 2-D correlation measurements in the search for the critical point-the point where the phase transition changes from a smooth crossover to first order phase transition.

The observation of an abrupt onset for the ridge provides an added motivation for conducting this energy scan. Several of the promising explanations for the formation of the ridge indicate that the onset of the ridge should coincide with the development of large pressure gradients. This increase in pressure at a particular energy density could be related to the QCD equation of state and therefore may be the most direct observation of the EOS accessible in heavy-ion collisions. The correct physical explanation for the ridge is still under debate - but having observed a long range correlation in heavy-ion collisions which shows an abrupt onset at a given energy density, the logical next step is to perform an energy scan. In this scan, the amplitude, longitudinal width, and azimuthal width of the ridge and critical density for ridge formation can be studied.

Acknowledgements I'd like to thank Raju Venugopalan and Adrian Dumitru for help with producing Fig. 3. I'd like to thank Ágnes Mócsy, Larry McLerran, Ron Longacre, and Sean Gavin for many helpful discussions.

\section{References}

1. G. Baym, G. Friedman, I. Sarcevic, Phys. Lett. B 219, 205 (1989)

2. K. Rajagopal, F. Wilczek, Nucl. Phys. B 399, 395 (1993). arXiv: hep-ph/9210253

3. G. Baym, H. Heiselberg, Phys. Lett. B 469, 7 (1999). arXiv:nuclth/9905022

4. S. Jeon, V. Koch, Phys. Rev. Lett. 85, 2076 (2000). arXiv:hep-ph/ 0003168 
5. F. Karsch, E. Laermann, A. Peikert, Phys. Lett. B 478, 447 (2000). arXiv:hep-lat/0002003

6. F. Karsch (RBC Collaboration and HotQCD Collaboration), arXiv:0804.4148 [hep-lat]

7. C. Schmidt, in Proc. CPOD2006 (2006), p. 002. arXiv:hep-lat/ 0701019

8. F. Karsch, in Proc. CPODO7 (2007), p. 026. arXiv:0711.0656 [hep-lat]

9. F. Karsch, in Proc. LAT2007 (2007), p. 015. arXiv:0711.0661 [hep-lat]

10. M. Daugherity (STAR Collaboration), arXiv:0806.2121 [nucl-ex]

11. J. Adams et al. (STAR Collaboration), Phys. Rev. Lett. 95, 152301 (2005)

12. F. Wang (STAR Collaboration), J. Phys. G 30, S1299 (2004). arXiv:nucl-ex/0404010

13. J. Adams et al. (STAR Collaboration), Phys. Rev. C 73, 064907 (2006). arXiv:nucl-ex/0411003

14. J. Putschke, J. Phys. G 34, S679 (2007). arXiv:nucl-ex/0701074

15. J. Adams et al. (STAR Collaboration), Phys. Rev. C 75, 034901 (2007). arXiv:nucl-ex/0607003

16. B. Srivastava (for the STAR Collaboration), Int. J. Mod. Phys. E 16, 3371 (2008)

17. B. Srivastava (STAR Collaboration), Feb. 4th-10th, 2008, to be published in conference proceedings

18. A. Adare et al. (PHENIX Collaboration), Phys. Rev. C 78, 014901 (2008). arXiv:0801.4545 [nucl-ex]

19. B. Alver et al. (PHOBOS Collaboration), arXiv:0804.3038 [nuclex]

20. A. Dumitru, F. Gelis, L. McLerran, R. Venugopalan, arXiv:0804. 3858 [hep-ph]

21. S. Gavin, L. McLerran, G. Moschelli, arXiv:0806.4718 [nucl-th]

22. T.A. Trainor (STAR Collaboration), AIP Conf. Proc. 828, 238 (2006). arXiv:nucl-ex/0510035

23. A. Majumder, B. Muller, S.A. Bass, Phys. Rev. Lett. 99, 042301 (2007). arXiv:hep-ph/0611135
24. S. Gavin, G. Moschelli, arXiv:0806.4366 [nucl-th]

25. S.A. Voloshin, Nucl. Phys. A 749, 287 (2005). arXiv:nucl-th/ 0410024

26. E.V. Shuryak, Phys. Rev. C 76, 047901 (2007). arXiv:0706.3531 [nucl-th]

27. N.K. Pruthi (STAR Collaboration), in Proceedings of ICHEP (2008, to appear)

28. P. Sorensen (STAR Collaboration), in Proc. CPOD2006 (2006), p. 019. arXiv:nucl-ex/0701028

29. T. Satogata et al. in Proc. CPOD2007 (2007), p. 051

30. J. Cleymans, H. Oeschler, K. Redlich, S. Wheaton, Phys. Rev. C 73, 034905 (2006). arXiv:hep-ph/0511094

31. F.R. Brown et al., Phys. Rev. Lett. 65, 2491 (1990)

32. A. Mocsy, F. Sannino, K. Tuominen, Phys. Rev. Lett. 92, 182302 (2004). arXiv:hep-ph/0308135

33. F. Karsch, M. Lutgemeier, Nucl. Phys. B 550, 449 (1999). arXiv:hep-lat/9812023

34. M. Asakawa, K. Yazaki, Nucl. Phys. A 504, 668 (1989)

35. A. Barducci, R. Casalbuoni, G. Pettini, R. Gatto, Phys. Rev. D 49, 426 (1994)

36. J. Berges, K. Rajagopal, Nucl. Phys. B 538, 215 (1999). arXiv: hep-ph/9804233

37. A.M. Halasz, A.D. Jackson, R.E. Shrock, M.A. Stephanov, J.J.M. Verbaarschot, Phys. Rev. D 58, 096007 (1998). arXiv: hep-ph/9804290

38. O. Scavenius, A. Mocsy, I.N. Mishustin, D.H. Rischke, Phys. Rev. C 64, 045202 (2001). arXiv:nucl-th/0007030

39. N.G. Antoniou, A.S. Kapoyannis, Phys. Lett. B 563, 165 (2003). arXiv:hep-ph/0211392

40. Y. Hatta, T. Ikeda, Phys. Rev. D 67, 014028 (2003). arXiv:hep-ph/ 0210284

41. M.A. Stephanov, Prog. Theor. Phys. Suppl. 153, 139 (2004). [Int. J. Mod. Phys. A 20, 4387 (2005)]. arXiv:hep-ph/0402115

42. M.A. Stephanov, K. Rajagopal, E.V. Shuryak, Phys. Rev. Lett. 81, 4816 (1998). arXiv:hep-ph/9806219 